\title{
Electro-Acupuncture on ST36 and SP6 Acupoints Ameliorates Lung Injury via Sciatic Nerve in a Rat Model of Limb Ischemia-Reperfusion
}

This article was published in the following Dove Press journal: Journal of Inflammation Research

\author{
Wei Lin' \\ Danyun Jia $\mathbb{D D}^{2}$ \\ Changchang $\mathrm{Fu}^{\prime}$ \\ Yihui Zheng' \\ Zhenlang Lin (D) \\ 'Department of Pediatrics, The Second \\ Affiliated Hospital and Yuying Children's \\ Hospital of Wenzhou Medical University, \\ Wenzhou, Zhejiang, 325000, People's \\ Republic of China; ${ }^{2}$ Department of \\ Anesthesiology, The First Affiliated \\ Hospital of Wenzhou Medical University, \\ Wenzhou, Zhejiang Province 325000 , \\ People's Republic of China
}

Correspondence: Zhenlang Lin Department of Pediatric

The Second Affiliated Hospital and Yuying Children's Hospital of Wenzhou Medical University, Wenzhou 325000, Zhejiang,

People's Republic of China

Email Izlprof2020@I63.com
Introduction: Electro-acupuncture (EA) can significantly improve inflammatory response, but the specific mechanism is not clear. Limb ischemia-reperfusion (I/R) first produces inflammatory reactions in the lungs. In this study, EA on Zusanli (ST36) and Sanyinjiao (SP6) were used to explore the mechanism of improving tissue inflammation by sciatic nerve disconnection.

Material and Methods: A total of 56 male Sprague-Dawley rats were randomly divided into sham group, model group, EA group, SEA group, SNC+EA group, TNC+EA group and PNC+EA group. The sham groups were not given any treatment. Rats in the model group were treated with limb I/R without acupuncture intervention. In the EA group, ST36 and SP6 were given EA treatment for $30 \mathrm{~min}$ before modeling. No electric current was given in the SEA group, and other operations were the same as those in the EA group. The SNC+EA group, TNC+EA group and PNC + EA group were respectively given sciatic nerve, tibial nerve or peroneal nerve amputation $72 \mathrm{~h}$ before modeling, and the others were the same as the model group.

Results: Compared with the sham group, $\mathrm{PaO} 2$ and a/A ratios decreased significantly in the model group ( $\mathrm{P}<0.05)$, while $\mathrm{PA}-\mathrm{aO} 2, \mathrm{RI}$, the ratio of wet to dry, lung injury value and inflammatory factor TNF- $\alpha$, IL-1, IL-6, and MPO increased significantly $(\mathrm{P}<0.05)$. Compared with the model group, $\mathrm{PaO} 2$, a/A ratios increased significantly in the EA group $(\mathrm{P}<0.05)$, while $\mathrm{PA}-\mathrm{aO} 2$, $\mathrm{RI}$, the ratio of wet to dry lung, lung injury value, and TNF- $\alpha$, IL-1, IL-6, and MPO decreased significantly (P $<0.05)$. After transection of the sciatic nerve, the protective effect of EA disappeared. However, when the peroneal or tibial nerve was severed, EA continued to maintain the protective effect.

Conclusion: EA on ST36 and SP6 can alleviate lung injury caused by limb I/R through the sciatic nerve.

Keywords: electro-acupuncture, limb ischemia-reperfusion injury, sciatic nerve

\section{Introduction}

Limb ischemia-reperfusion injury $(\mathrm{I} / \mathrm{R})$ is common during trauma, surgery and vascular embolism. ${ }^{1}$ After reperfusion injury, harmful substances can endanger distal organs through the bloodstream. The lung is highly sensitive to such inflammatory responses and is prone to acute injury during reperfusion. ${ }^{2,3}$ This damage process is usually caused by a variety of complex mechanisms, including inflammatory response, oxidative stress and immune dysfunction. ${ }^{3,4}$

Acupuncture has long been used in China as a traditional treatment. Electroacupuncture (EA) is a non-invasive procedure based on acupuncture. ${ }^{5}$ Studies have shown that EA pretreatment has anti-inflammatory effects on lung injury caused by inflammatory diseases, but the specific mechanism of EA's effect is still unclear. ${ }^{6}$ In 
this study, the limb ischemia-reperfusion model was used to observe the pulmonary inflammation and to investigate whether EA could also reduce the lung injury caused by limb I/R by inhibiting the inflammatory response. Meanwhile, sciatic nerve, tibial nerve and peroneal nerve were cut to explore whether the anti-inflammatory effect of EA was related to nerve.

\section{Methods}

\section{Animals}

Male Sprague-Dawley (SD) rats were used as research objects in this study. The S-D rats were 7-8 weeks old and weighed 250-300 grams. The rats were kept in incubators at $24^{\circ} \mathrm{C}$ for 12 hours on a light-dark cycle and were given standard food and running water. All animal care and experiments were conducted in strict accordance with the Guidelines for the Care and Use of Laboratory Animals by the National Institutes of Health and approved by the Laboratory Animal Ethics Committee of Wenzhou Medical University. The number of the use of Laboratory Animal is SYXK (Zhejiang) 2015-0009.

\section{Modelling}

Fifty-six rats were randomly divided into 7 groups ( 8 rats in each group). The sham group did not operate. The model group $^{7,8}$ had posterior limb ischemia for 3 hours and reperfusion for 3 hours (I/R), and there was no medication or EA treatment. In the EA group, the rats received EA for 30 minutes immediately before I/R, and the 2 and $15 \mathrm{~Hz}$ diffusion waves with stimulation current parameters of $1 \mathrm{~mA}$. In the SEA group, Electro-acupuncture at Zusanli (ST36) and Sanyinjiao (SP6) was 2-3 mm deep, without current stimulation. In the sciatic nerve detachment ( $\mathrm{SNC}+\mathrm{EA}$ ) group, tibial nerve detachment (TNC+EA) group and the peroneal nerve detachment (PNC+EA) group, sciatic nerve, radial nerve and peroneal nerve were cut, respectively, 72 hours before EA
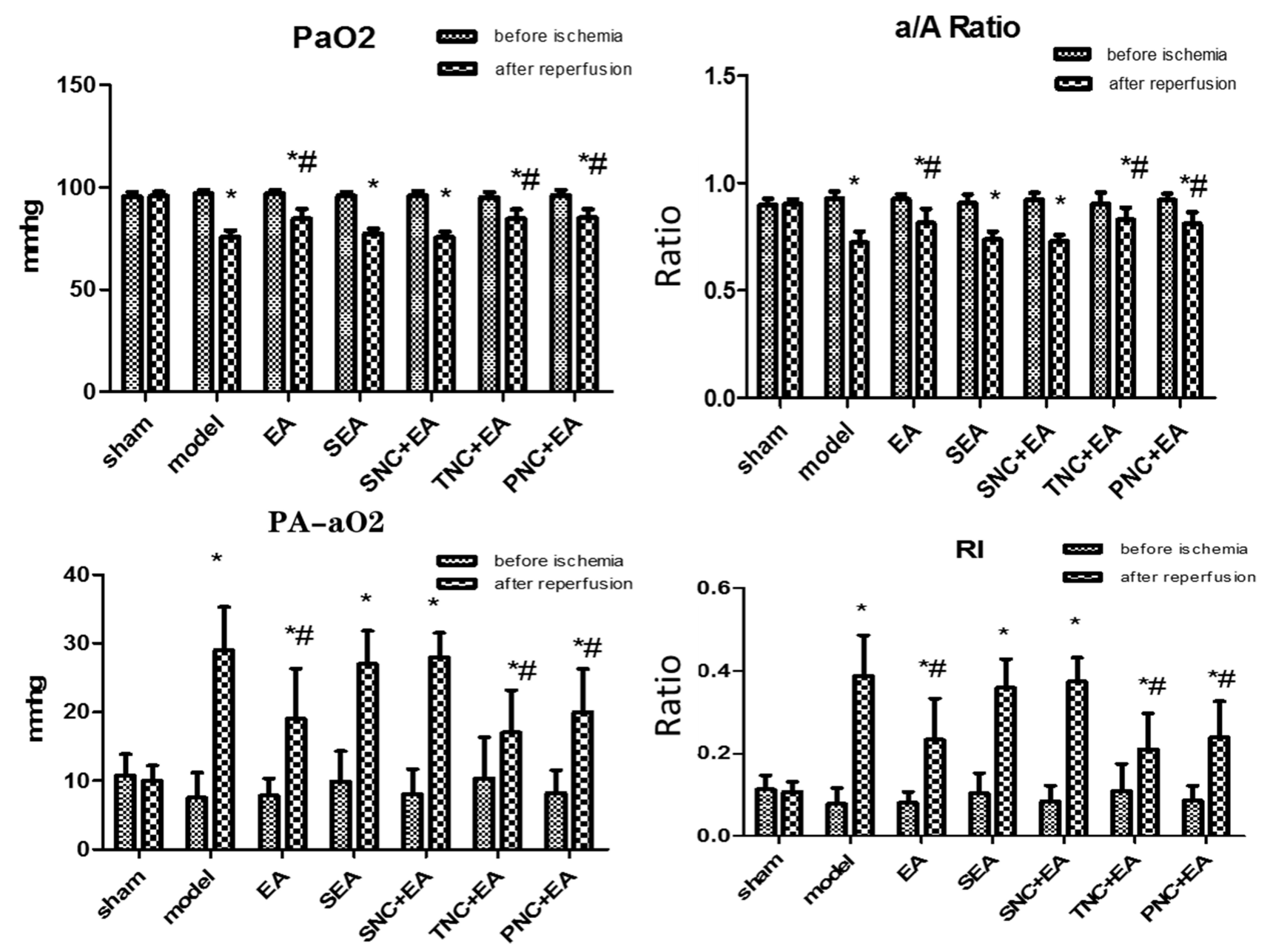

Figure I Blood gas analysis parameters $(\mathrm{PaO} 2, \mathrm{PA}-\mathrm{aO} 2, \mathrm{a} / \mathrm{A}$ ratio, and $\mathrm{RI})$ were measured before and after modeling. Results are presented as mean $\pm \mathrm{SEM}(\mathrm{n}=8$ rats per group). ${ }^{*} P<0.05$, versus the sham group; $\# P<0.05$, versus the model group. 


\section{Wet/dry weight ratio}

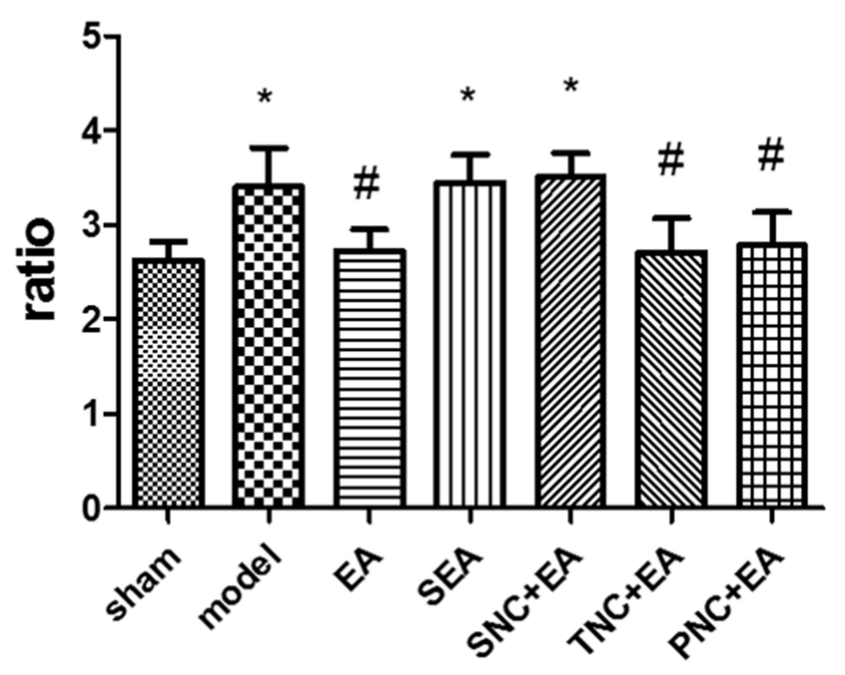

Figure 2 Wet-to-dry weight ratios for the groups. Results are presented as mean \pm SEM ( $n=8$ rats per group). $* P<0.05$, versus the sham group; $\# P<0.05$, versus the model group.

pretreatment, and $2 \mathrm{~mm}$ from the bifurcation point was selected as the cutting point. The experimental operation was the same as that in EA group.

\section{Blood Gas Analysis}

The content of oxygen partial pressure of arterial blood $(\mathrm{PaO} 2)$ and carbon dioxide partial pressure of arterial blood (PaCO2) were determined by blood gas analysis of the left common carotid artery. Data obtained by calculation include artery-alveolar oxygen partial pressure ratio $(\mathrm{a} / \mathrm{A})$, alveolar oxygen partial pressure difference (PA-aO2) and respiratory index (RI). The $\mathrm{PaO} 2$ reflects cardiopulmonary function and the degree of hypoxia. The $\mathrm{PaCO} 2$ reflects alveolar ventilation. The a/A, $\mathrm{PA}-\mathrm{aO} 2$ and $\mathrm{RI}$ reflect Pulmonary gas exchange function.

\section{Wet/Dry Weight Ratio of Lung}

The upper right lung tissue of each rat was weighed (wet weight) immediately after removal. The slices were dried in an oven at $60^{\circ} \mathrm{C}$ for 2 days until they reached a steady dry weight. Then, calculate the wet-dry weight ratio by dividing the wet weight by the dry weight.

\section{HE Staining and Lung Injury Score}

The lungs were perfused with $4 \%$ paraformaldehyde after cold saline infusion, fixed in 4\% paraformaldehyde for 24 hours at $4^{\circ} \mathrm{C}$, then embedded in paraffin tissue, cut into $5 \mu \mathrm{m}$ thick slices, and then stained with hematoxylin and eosin. Lung injury scores were based on four independent parameters: alveolar edema, hemorrhage, infiltration of inflammatory cells, and thickened alveolar septum.

\section{Inflammatory Factor and MPO Concentrations in the Rat Lung}

Concentrations of IL-1 $\beta$, IL-6, TNF- $\alpha$, and MPO in rat lungs were determined by ELISA kit (Boyun Co., Shanghai, China). Absorbance values were read using
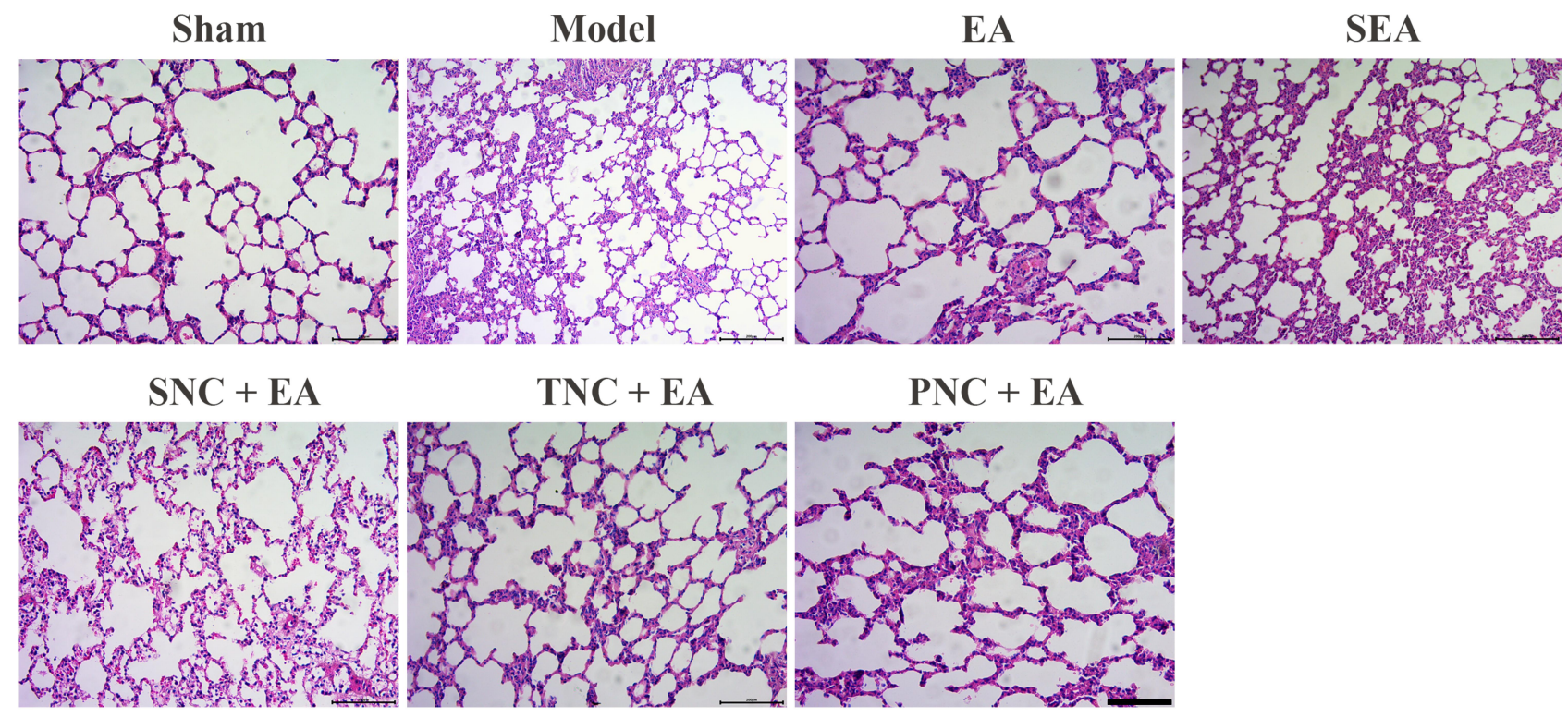

Figure 3 Hematoxylin-eosin (H\&E) staining and lung injury. Representative photomicrographs of lung sections stained with H\&E in the lungs of the rats in all seven groups. Scale bar $=200 \mu \mathrm{m}$. 


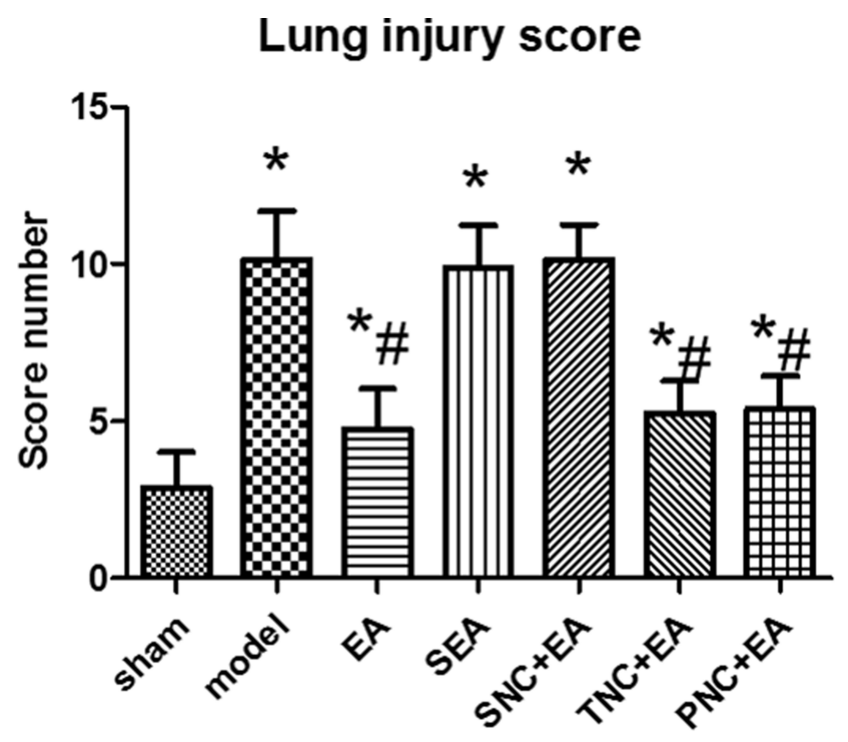

Figure 4 The lung injury scores of the rats in all seven groups. Results are presented as mean \pm SEM ( $n=8$ rats per group). $* p<0.05$, versus the sham group; $\# P<0.05$, versus the model group. a multi-function microplate reader (Thermo Fisher Scientific, Waltham, MA, USA).

\section{Statistical Analyses}

Numerical data were expressed as the mean \pm standard deviation. After LSD test, statistical significance was determined by one-way analysis of variance (ANOVA) when analyzing more than two groups. $\mathrm{P}<0.05$ was considered significant. Social science software statistical programs (SPSS19.0) were used for analysis.

\section{Results}

\section{Blood Gas Analysis}

As shown in Figure 1, the parameters of blood gas analysis $(\mathrm{PaO} 2, \mathrm{PA}-\mathrm{aO} 2, \mathrm{a} / \mathrm{A}$ ratio and $\mathrm{RI})$ in the 7 groups before ischemia were not statistically significant. However, after ischemia for 3 hours and then reperfusion for 3 hours in
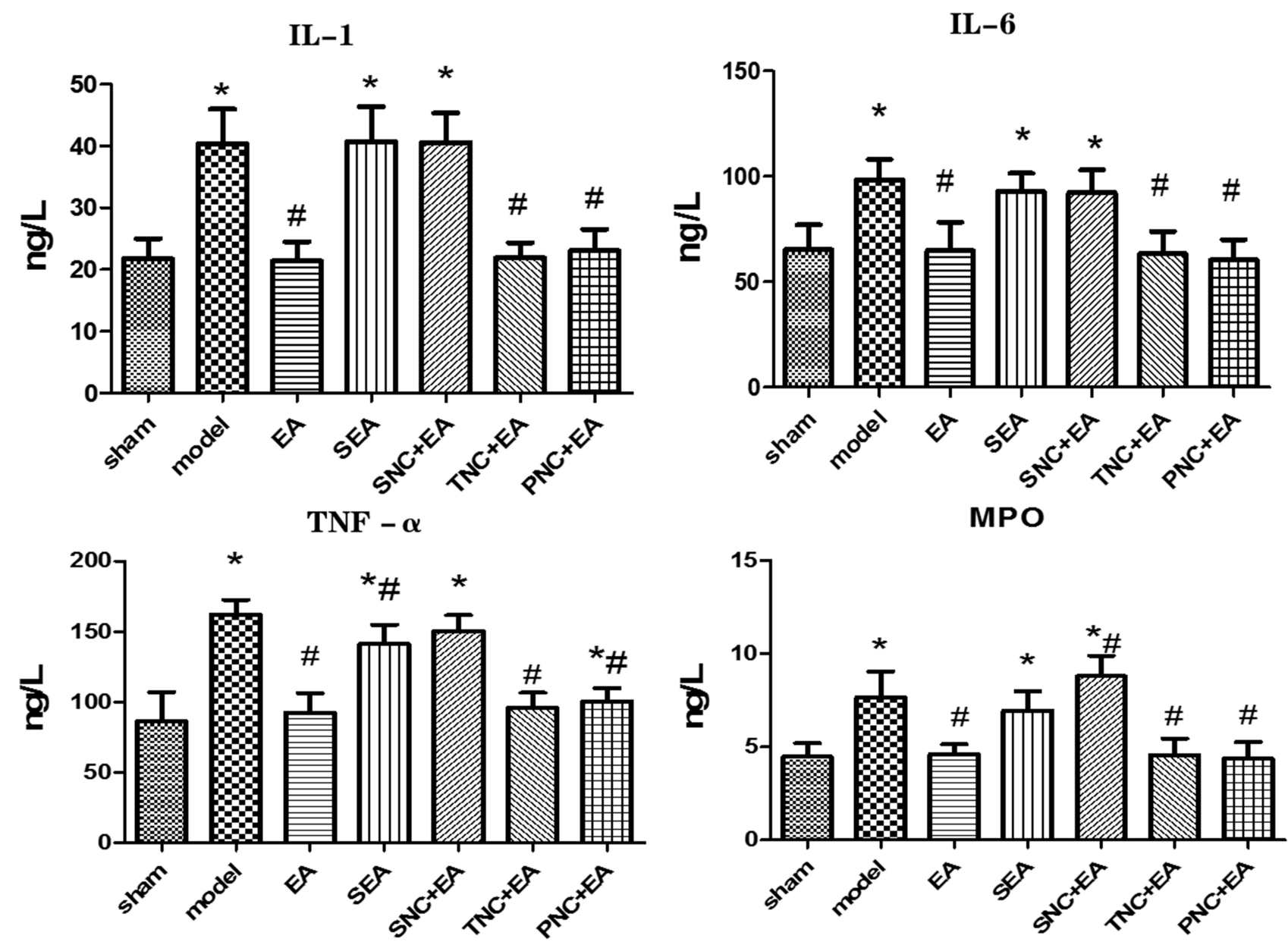

Figure 5 Inflammatory cytokines and MPO. IL-I, IL-6, TNF- $\alpha$, and MPO expression levels were measured in the lungs of the rats in all seven groups. Results are presented as mean $\pm \operatorname{SEM}(\mathrm{n}=8$ rats per group). $* P<0.05$, versus the sham group; $\# P<0.05$, versus the model group. 
the model group, the $\mathrm{PaO} 2$ and $\mathrm{a} / \mathrm{A}$ ratio were significantly reduced, whereas the $\mathrm{PA}-\mathrm{aO} 2$ and $\mathrm{RI}$ were significantly increased $(\mathrm{P}<0.05)$. Compared with the model group, $\mathrm{PaO} 2$ and $\mathrm{a} / \mathrm{A}$ ratio increased in the EA group, TNC + $\mathrm{EA}$ group and $\mathrm{PNC}+\mathrm{EA}$ group $(\mathrm{P}<0.05)$, while $\mathrm{PA}-\mathrm{aO} 2$ and $\mathrm{RI}$ decreased $(\mathrm{P}<0.05)$. There was no significant difference in $\mathrm{PaO} 2, \mathrm{a} / \mathrm{A}$ ratio, $\mathrm{PA}-\mathrm{aO} 2$ and $\mathrm{RI}$ between $\mathrm{SNC}+\mathrm{EA}$ group and model group $(\mathrm{P}>0.05)$.

\section{Wet-to-Dry Weight Ratio}

As shown in Figure 2. Compared with the sham group, the wet to dry weight ratio increased in the model group. EA pre-treatment can reduce the ratio of wet-to-dry weight ( $\mathrm{P}$ $<0.05$ ), but its effect may be blocked by sciatic nerve transection. Compared with the model group, the wet to dry ratio in the TNC + EA group and $\mathrm{PNC}+\mathrm{EA}$ group decreased $(\mathrm{P}<0.05)$.

\section{Hematoxylin and Eosin Staining and Lung Injury}

Figures 3 and 4 show lung interstitial edema and alveolar structural destruction in the model group. Compared with the model group, the EA group had less lung damage. The TNC+EA group and PNC+EA group had lower lung injury scores compared with the model group $(\mathrm{P}<0.05)$. There was no significant difference in the lung injury score between SNC+EA group and model group $(\mathrm{P}>0.05)$.

\section{Inflammatory Cytokines and MPO}

As shown in Figure 5, compared with the sham group, the model and SNC+EA group had higher levels of inflammatory cytokines (IL-1, IL-6, TNF- $\alpha$, and MPO) $(\mathrm{P}<0.05)$. Compared with the model group, the EA group, TNC+EA group and PNC+EA group demonstrated lower levels of inflammatory cytokines and MPO $(\mathrm{P}<0.05)$.

\section{Discussion}

In this study, EA pretreatment at ST36 and SP6 for 30 minutes significantly reduced the inflammatory responses as well as the release of inflammatory cytokines in the lungs of $\mathrm{I} / \mathrm{R}$ injured rats. However, the specific mechanism of lung injury after limb ischemia-reperfusion remains unclear, which may be related to the release of inflammatory cytokines caused by limb $\mathrm{I} / \mathrm{R}$, the enhancement of oxidative stress, the extensive release of oxygen-free radicals, and the occurrence of apoptosis during reperfusion. ${ }^{8-}$ ${ }^{10}$ It is also possible that limb I/R induces the activation of neutrophils and monocytes/macrophages, promoting inflammatory responses and releasing large amounts of inflammatory mediators, including cytokines and chemokines. It was found in the physical experiment that cytokines, TNF- $\alpha$, IL- 1 , and IL- 6 were significantly changed when I/R was applied to limbs. ${ }^{10-12}$ Therefore, the occurrence of acute lung injury is closely related to the activation of inflammatory factors.

During lung injury, TNF- $\alpha$ is the most important inflammatory cytokine produced by alveolar cells, which can activate polymorphonuclear white blood cells in the blood and cause them to accumulate at the site of alveolar injury. ${ }^{13,14} \mathrm{TNF}-\alpha$ can activate inflammatory cells and endothelial cells, increase the expression of IL-1, IL-6, IL-8, thus promoting the degranulation of polymorphonuclear leukocytes, lysosome release, mediating alveolus-capillary membrane injury, and destroying the barrier function of the inflammatory cascade. ${ }^{15} \mathrm{IL}-1$ has a strong chemotactic effect, and can induce the expression of vascular endothelial cell adhesion molecules, thus promoting leukocyte adhesion. ${ }^{16}$ IL-6 is an important cytokine involved in the systemic immune response and the inflammatory cascade, which activates neutrophils and increases the production of inflammatory mediators. ${ }^{17} \mathrm{MPO}$ is produced by neutrophil white blood cells and can reflect the activation of polymorphonuclear white blood cells in the lung. ${ }^{18}$ Inflammatory cytokines are released throughout the body after limb I/R, especially in lung tissue. In this study, it was found the expressions of IL-1, IL-6, IL-8, and TNF - $\alpha$ were significantly increased after modeling, accompanied by a decline in pulmonary ventilation function.

As a non-invasive intervention, EA has significant antiinflammatory effects on many diseases. ${ }^{19,20}$ Studies have shown that electrical stimulation of Zusanli and Quchi can reduce the level of inflammatory factors after reperfusion injury in rats with middle cerebral artery occlusion. ${ }^{21}$ At the same time, EA has been found to have a protective effect on lung injury in animals. ${ }^{22}$ In this study, the EA group can significantly reduce the expression of inflammatory factors, and significantly improve the lung ventilation function and lung injury. The protective effect of EA pretreatment disappeared in the SNC+EA group, but maintained in the tibial nerve detachment TNC+EA group and the peroneal nerve detachment PNC+EA group. So EA pretreatment of Zusanli and Sanyinjiao may carry out an anti-inflammatory effect through the sciatic nerve.

However, this study also has some limitations. We have only found that the anti-inflammatory effects of electro-acupuncture on Zusanli and Sanyinjiao may be related 
to the sciatic nerve, but the exact mechanism is unclear. More work need to be done to examine the molecular aspects and pathway of EA in order to explore the mechanism through which the sciatic nerve works.

\section{Funding}

This research did not receive any specific grant from funding agencies in the public, commercial, or not-forprofit sectors.

\section{Disclosure}

The authors declare that they have no conflict of interests for this work.

\section{References}

1. Olinic DM, Stanek A, Tataru DA, et al. Acute limb ischemia: an update on diagnosis and management. J Clin Med. 2019;8:8.

2. Zou R, Wang MH, Chen Y, et al. Hydrogen-rich saline attenuates acute lung injury induced by limb ischemia/reperfusion via down-regulating chemerin and NLRP3 in rats. Shock. 2019;52(1):134-141. doi:10.1097/SHK.0000000000001194

3. Bergmann A, Jovanovska E, Schilling T, et al. Early and late effects of remote ischemic preconditioning on spirometry and gas exchange in healthy volunteers. Respir Physiol Neurobiol. 2019;271:103287.

4. Chen $\mathrm{K}, \mathrm{Xu} \mathrm{Z}$, Liu Y, et al. Irisin protects mitochondria function during pulmonary ischemia/reperfusion injury. Sci Transl Med. 2017;9(418):eaao6298. doi:10.1126/scitranslmed.aao6298

5. Chon TY, Lee MC. Acupuncture. Mayo Clin Proc. 2013;88 (10):1141-1146. doi:10.1016/j.mayocp.2013.06.009

6. Wang Z, Chen T, Long M, et al. Electro-acupuncture at Acupoint ST36 ameliorates inflammation and regulates Th1/Th2 balance in delayed-type hypersensitivity. Inflammation. 2017;40(2):422-434. doi:10.1007/s10753-016-0487-z

7. Dick F, Li J, Giraud MN, et al. Basic control of reperfusion effectively protects against reperfusion injury in a realistic rodent model of acute limb ischemia. Circulation. 2008;118(19):1920-1928. doi:10.1161/ CIRCULATIONAHA.108.787754

8. Welbourn R, Goldman G, Kobzik L, et al. Role of neutrophil adherence receptors (CD 18) in lung permeability following lower torso ischemia. Circ Res. 1992;71(1):82-86. doi:10.1161/01.RES.71.1.82

9. Klausner JM, Paterson IS, Goldman G, et al. Thromboxane A2 mediates increased pulmonary microvascular permeability following limb ischemia. Circ Res. 1989;64(6):1178-1189. doi:10.1161/01. RES.64.6.1178
10. Shih YM, Shih JM, Hou YC, et al. Pretreatment with fish oil-based lipid emulsion modulates muscle leukocyte chemotaxis in murine model of sublethal lower limb ischemia. Mediators Inflamm. 2017;2017:4929346.

11. Mo Y, Chen S, Yang L, et al. The effect of transcutaneous electrical acupoint stimulation on inflammatory response in patients undergoing limb ischemia-reperfusion. Mediators Inflamm. 2017;2017:8369737.

12. Zhao YR, Lv WR, Zhou JL. Role of carbonyl sulfide in acute lung injury following limb ischemia/reperfusion in rats. Eur $J$ Med Res. 2017;22(1):12. doi:10.1186/s40001-017-0255-z

13. Ware LB. Prognostic determinants of acute respiratory distress syndrome in adults: impact on clinical trial design. Crit Care Med. 2005;33(3 Suppl):S217-S222. doi:10.1097/01. CCM.0000155788.39101.7E

14. Bruder D, Srikiatkhachorn A, Enelow RI. Cellular immunity and lung injury in respiratory virus infection. Viral Immunol. 2006;19 (2):147-155. doi:10.1089/vim.2006.19.147

15. Verhelst K, Carpentier I, Beyaert R. Regulation of TNF-induced NF-kappaB activation by different cytoplasmic ubiquitination events. Cytokine Growth Factor Rev. 2011;22(5-6):277-286. doi:10.1016/j.cytogfr.2011.11.002

16. Brown Z, Strieter RM, Neild GH, et al. IL-1 receptor antagonist inhibits monocyte chemotactic peptide 1 generation by human mesangial cells. Kidney Int. 1992;42(1):95-101. doi:10.1038/ ki. 1992.266

17. Garbers C, Heink S, Korn T, et al. Interleukin-6: designing specific therapeutics for a complex cytokine. Nat Rev Drug Discov. 2018;17 (6):395-412. doi:10.1038/nrd.2018.45

18. Yang CW, Liu H, Li XD, et al. Salvianolic acid B protects against acute lung injury by decreasing TRPM6 and TRPM7 expressions in a rat model of sepsis. J Cell Biochem. 2018;119(1):701-711. doi: $10.1002 /$ jcb. 26233

19. Salazar TE, Richardson MR, Beli E, et al. Electro-acupuncture promotes central nervous system-dependent release of mesenchymal stem cells. Stem Cells. 2017;35(5):1303-1315. doi:10.1002/ stem. 2613

20. Zhan J, Qin W, Zhang Y, et al. Upregulation of neuronal zinc finger protein A20 expression is required for electro-acupuncture to attenuate the cerebral inflammatory injury mediated by the nuclear factor-kB signaling pathway in cerebral ischemia/reperfusion rats. $J$ Neuroinflammation. 2016;13(1):258. doi:10.1186/s12974-0160731-3

21. Weilin L, Xian, et al. Electroacupuncture inhibits inflammatory injury by targeting the miR-9-mediated NF- $\mathrm{kB}$ signaling pathway following ischemic stroke. Mol Med Rep. 2016.

22. Jianbo Y, Shuan D, Xiaoqing L, et al. Role of HO-1 in protective effect of electro-acupuncture against endotoxin shock-induced acute lung injury in rabbits. Exp Biol Med. 2013;238(6):705-712. doi:10.1177/1535370213489487
Journal of Inflammation Research

\section{Publish your work in this journal}

The Journal of Inflammation Research is an international, peerreviewed open-access journal that welcomes laboratory and clinical findings on the molecular basis, cell biology and pharmacology of inflammation including original research, reviews, symposium reports, hypothesis formation and commentaries on: acute/chronic inflammation; mediators of inflammation; cellular processes; molecular mechanisms; pharmacology and novel anti-inflammatory drugs; clinical conditions involving inflammation. The manuscript management system is completely online and includes a very quick and fair peerreview system. Visit http://www.dovepress.com/testimonials.php to read real quotes from published authors. 